\title{
Chlamydia trachomatis Alters Iron-Regulatory Protein-1 Binding Capacity and Modulates Cellular Iron Homeostasis in HeLa-229 Cells
}

\author{
Harsh Vardhan, Apurb R. Bhengraj, Rajneesh Jha, and Aruna Singh Mittal \\ Division of Microbiology/Tissue Culture, Institute of Pathology, Indian Council of Medical Research (ICMR), \\ Safdarjung Hospital Campus, New Delhi, Pin 110029, India \\ Correspondence should be addressed to Aruna Singh Mittal, amittal_cp@rediffmail.com
}

Received 26 March 2009; Accepted 8 June 2009

Recommended by Mark Smith

Chlamydia trachomatis (CT) is the leading cause of diseases related to reproductive health and iron plays important role in chlamydial pathogenesis. Iron homeostasis in chlamydia-infected cells is not clear thus far. This study shows that expression of the transferrin receptor (TfR) is downregulated, whereas expression of the ferritin heavy chain is upregulated in CT-infected HeLa-229 cells. Expression of iron-regulatory protein (IRP)-1 predominates over IRP-2 in infected cells. In infected cells, attenuated binding activity of IRP-iron responsive elements (IREs) is observed using the electrophoretic mobility-shift assay. These results suggest that iron homeostasis is modulated in CT-infected HeLa cells at the interface of acquisition and commensal use of iron.

Copyright (C) 2009 Harsh Vardhan et al. This is an open access article distributed under the Creative Commons Attribution License, which permits unrestricted use, distribution, and reproduction in any medium, provided the original work is properly cited.

\section{Introduction}

Chlamydia trachomatis (CT) is one of the most successful and leading bacterial pathogens that cause sexually transmitted infections; it infects and grows within the genital mucosal epithelial cells [1]. Chlamydial infection is responsible for pelvic inflammatory disease, tubal infertility, ectopic pregnancy, and endometritis, having significant impact on the reproductive health of women worldwide [2]. The developmental cycle of $C T$ includes two forms: an infectious elementary body (EB) and a reticulate body that multiplies within the inclusion by binary fission [3]. A third developmental form-the persistent form and is considered as a mechanism of survival under stressful conditions $[4,5]$. Persistence is induced in response to changes in the culture medium, including amino acid or iron deprivation and in the presence of antibiotics or cytokines such as gamma interferon (IFN- $\gamma)$ [6-10].

Iron is the most essential element because of its role in the chemistry related to bioenergetics, being involved in a myriad of biochemical events that are required for growth and development [11]. Excess iron promotes the generation of reactive radicals, which channelize pathogenic cascades that damage cells and tissues. Iron, as an essential nutrient and potential toxin, poses an exquisite regulatory problem in biological systems [12]. Iron homeostasis of cells is regulated posttranscriptionally by the binding of iron-regulatory proteins (IRP)-1 and -2 with iron-responsive elements (IREs). In iron-starved cells, binding of IRP to IRE stabilizes the transferrin-receptor (TfR) mRNA and inhibits the translation of mRNAs that encode the heavy $(\mathrm{H})$ and light (L) chains of ferritin, thus promoting cellular iron uptake and preventing iron sequestration [13].

Almost all cells and organisms exploit iron as a cofactor in a multitude of biochemical activities. CT EBs harvested from desferal-exposed polarized epithelial cells has a reduced infective potential, which is restored after replacement with iron-saturated transferrin medium, thus confirming the dependence of chlamydia on the host for its requirement of iron [14]. Chlamydial genomic annotation provides an idea about the iron-transporter analog of ExbB/D; however, an identifiable TonB is absent [15]. The CT divalent cationdependent regulator is a distant relative of the ferric-uptake regulator family of iron-responsive regulators [16]. The biphasic life cycle of chlamydia provides various opportunities for cross-communication with the host regulatory 
network. No study is available currently regarding the involvement of chlamydiae in the regulation of intracellular iron in infected HeLa cells. This study aims to provide a clue to the chlamydial modulatory effect on host cells at the interface of acquisition and commensal use of iron.

\section{Materials and Methods}

Unless otherwise stated, all the reagents were purchased from Sigma Aldrich (Saint Louis, Mo, USA) and antibodies, from Santa Cruz Biotechnology (Santa Cruz, Calif, USA). Plastic wares and glasswares for tissue culture were obtained from Greiner, Germany.

2.1. Culture of CT. CT, laboratory reference serovar D (D/UW-3/Cx), propagated in HeLa-229 cells as described previously [17], were purified and stored at $-80^{\circ} \mathrm{C}$ in sucrose-phosphate-glutamate medium ( $\mathrm{pH} 7.0)$. The inocula were confirmed to be free from contamination with Mycoplasma spp. using specific kits (Mycoplasma detection kit, Takara, Madison, USA).

2.2. Infection Protocol. HeLa-229 $\left(1 \times 10^{6}\right.$ cells/well $)$ cells were seeded in 6-well tissue-culture plates (Greiner, Germany). Subsequently, the HeLa cell monolayer was washed with Hank's balanced salt solution (HBSS) and inoculated with chlamydial EBs at a multiplicity of infection (moi) of 2. Next, the tissue-culture plates were placed on a rocker (Bangalore Genei Pvt. Ltd, Bangalore, India) for 2 hours at $35^{\circ} \mathrm{C}$ to obtain a homogeneous infectivity. The infected monolayer was washed twice with HBSS and supplemented with complete Dulbecco's modified eagle medium containing $10 \%$ fetal calf serum. Infected HeLa cells were incubated at $37^{\circ} \mathrm{C}$ with $5 \% \mathrm{CO}_{2}$ in a humid environment. Deferoxamine (DFX) and ferric ammonium citrate (FAC) were added after 16 hours postinfection (hpi) in the respective wells; after $24 \mathrm{hpi}$, the cells were withdrawn for analysis. All the experiments were carried out in triplicate with mock-infected cells as their respective controls.

2.3. Cytosolic Extract Preparation. Infected $\left(2 \times 10^{6}\right)$ cells were lysed with $140 \mu \mathrm{L}$ of lysis buffer $(20 \mathrm{mM} \mathrm{N}-2$ hydroxyethylpiperazine- $\mathrm{N}^{\prime}$-2-ethanesulfonic acid (HEPES), $25 \mathrm{mM} \mathrm{KCl}, 0.5 \% \mathrm{NP}-40$, and $1 \mathrm{mM} \mathrm{DTT}$ ) for 10 minutes on ice. Subsequently, the lysates were centrifuged (Platocraft, Rota $4 \mathrm{R}-\mathrm{V}$, Mumbai, India) at $15,000 \times \mathrm{g}$ for 15 minutes at $4^{\circ} \mathrm{C}$ to remove the cell debris and nucleus. Protein concentrations were determined by the Bradford protein assay (BioRad Laboratories, USA), with bovine serum albumin (BSA) as the standard.

2.4. Electrophoretic Mobility-Shift Assay. A radiolabeled probe of the IRE was transcribed by the in vitro transcription method. The IRE sequence of 5'GAGTTCCGTCCAAGCACUTGAAGCAGGAAACTCTCTCCCTATAGTGAGTCGTATTA3' was used as the template, and the T7 sequence $5^{\prime}$ TATAGTGAGTCGTATTA3' as the primer for the amplification step carried out using an in vitro transcription kit (Roche Applied Science, Penzberg, Germany). The probe was treated with RNase-free DNase I to remove DNA contamination and subsequently extracted by the phenol-chloroform (1 : 1, v/v) method. The cytoplasmic extract $(20 \mu \mathrm{g})$ was mixed with $10 \mathrm{ng}$ of $\left[{ }^{32} \mathrm{P}\right]$ CTP-labeled IRE in binding buffer (10 mM HEPES, pH 7.6, $40 \mathrm{mM}$ $\mathrm{KCl}, 3 \mathrm{mM} \mathrm{MgCl}_{2}$, and $5 \%$ glycerol) and incubated at room temperature for 20 minutes. After incubation, the complex was resolved on a $6 \%$ nondenaturing acrylamide gel. In a parallel experiment, samples were treated with 2-mercaptoethanol (2-ME) at a final concentration of $2 \%$ before addition of the IRE probe, to allow the complete expression of IRE-binding activity. Amersham phosphoscreen was exposed to the gels for autoradiography and scanned using a PhosphorImager (Typhoon 9700, Amersham, Uppasala, Sweden). Band-shift experiments were carried out at least three times, and one representative experiment is shown.

2.5. Immunoblotting of Proteins. CT-infected HeLa cells were washed with phosphate buffered saline (PBS) and subsequently treated with lysis buffer (0.5\% Nonidet P40, $150 \mathrm{mM} \mathrm{NaCl}, 0.1 \%$ sodium dodecyl sulfate (SDS), $50 \mathrm{mM} \mathrm{NaF}, 1 \mathrm{mM} \mathrm{Na} \mathrm{VO}_{4}$, and $1 \mathrm{mM}$ phenyl methyl sulfonyl fluoride) containing the complete protease-inhibitor cocktail (Roche Diagnostics, Mannheim, Germany). Protein concentrations were determined by the Bradford protein assay (BioRad Laboratories) with BSA as standard. Extracted proteins $(40 \mu \mathrm{g})$ were electrophoresed on $8-12 \%$ SDSpolyacrylamide gels and transferred to polyvinyl difluoride membranes (BioRad Laboratories); the membranes were reversibly stained with Ponceau S (Sigma Aldrich) to confirm complete transfer. Membranes were blocked with 5\% nonfat dry milk in PBS-Tween-20 and incubated with rabbit antiIgGs against TfR-1, ferritin heavy chain (FHC), IRP-1, and IRP-2; they were further incubated with the goat anti-rabbit IgG conjugated with horseradish peroxidase. Subsequently, they were developed using diaminobenzamide as the detection agent and analyzed using the Image J software.

2.6. Statistical Analysis. Statistical analysis was carried out with the GraphPad Prism software (version 5.0). Differences were tested for statistical significance by one-way analysis of variance, followed by the Bonferroni posttest. Each experiment was carried out in triplicate.

\section{Results}

3.1. Expression of TfR in CT-Infected HeLa Cells. As visualized by the Western blot assay, expression of TfR decreased in CT-infected HeLa cells in comparison to mock-infected cells. After addition of the intracellular iron chelator DFX, there was no change in the expression of TfR, whereas mock infection showed a significant increase. However, iron supplementation with FAC to $C T$-infected HeLa cells did not lead to a change in the TfR level, in comparison to the respective controls (Figure $1(\mathrm{a})$ ). 

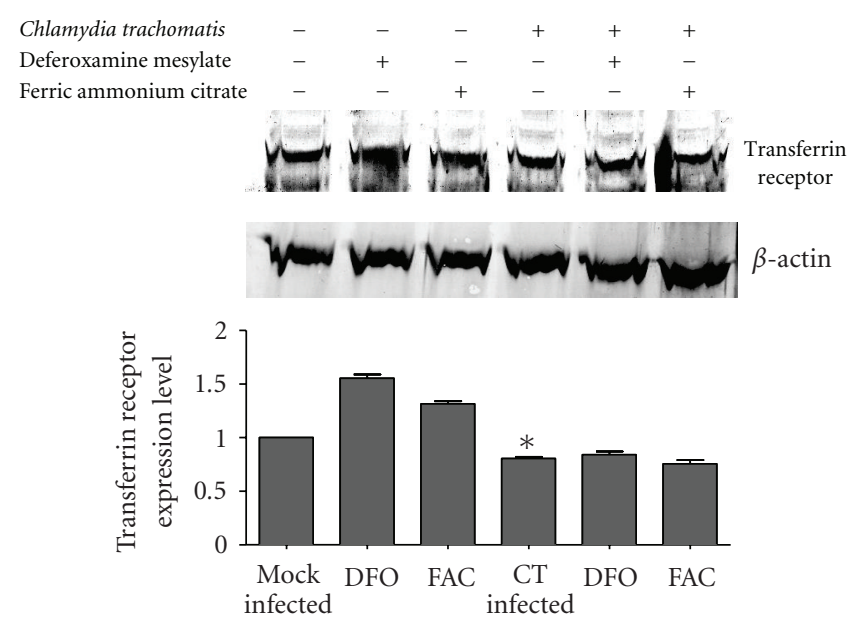

(a)

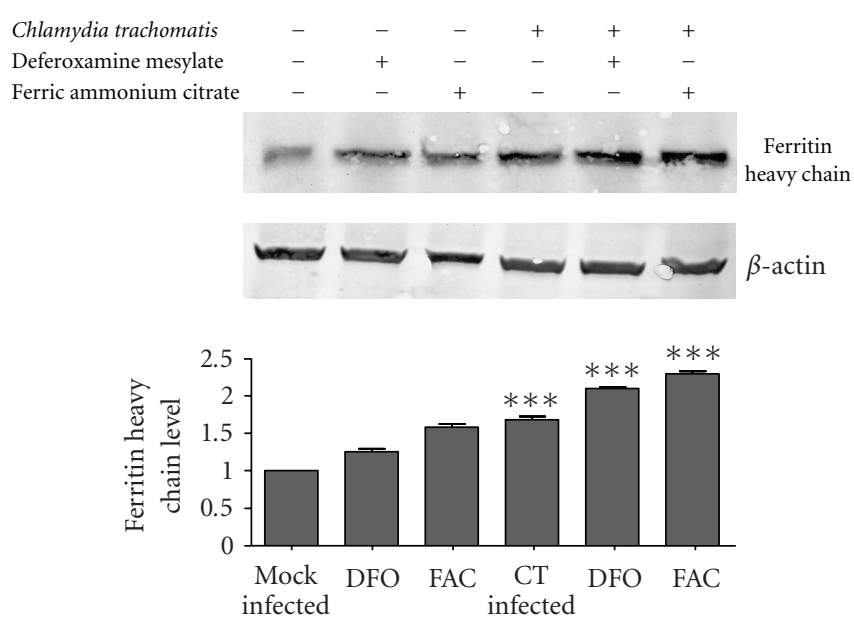

(b)

FIgURE 1: Effect of CT infection on TfR expression in HeLa cells: HeLa cells were infected at 2 moi and treated with the iron chelator DFX mesylate and ferric ammonium citrate (FAC). Mock-infected cells were considered as the control. Treated and untreated cells were harvested at $24 \mathrm{hpi}$, and the cell lysates $(40 \mu \mathrm{g})$ were electrophoresed on $8-12 \%$ SDS-PAGE gel, followed by Western blot analysis using anti-TfR and anti-FHC antibodies, with beta-actin as the loading control. (a) Expression of the TfR was downregulated in CT-infected HeLa cells and did not change after addition of DFX and FAC. (b) Expression of FHC was studied in CT-infected HeLa cells in comparison to control. FHC level remained above the basal level (mock) in CT-infected cells after addition of DFX. Conversely, addition of FAC led to a higher degree of induction in the FHC level of CT-infected cells than that in the control. Bars represent standard error. ${ }^{*} P<.05$ and ${ }^{* *} P<.01$.

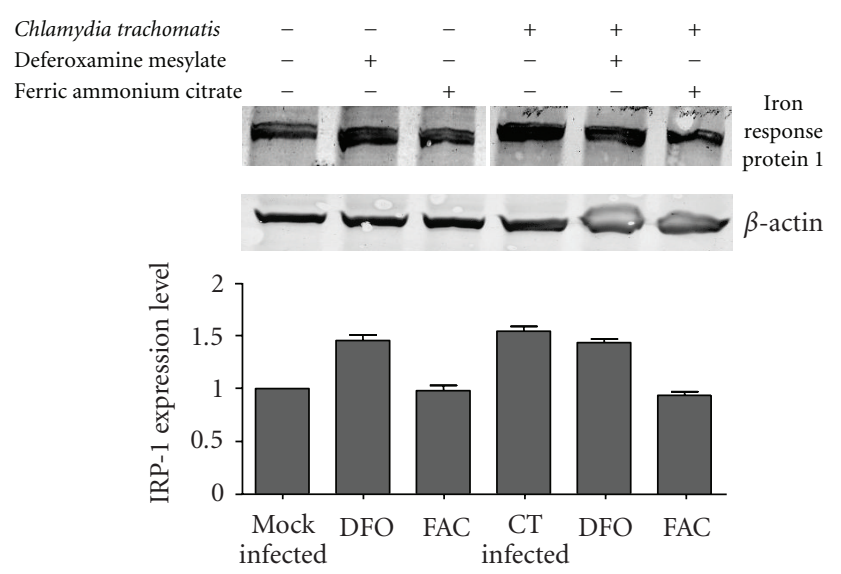

(a)

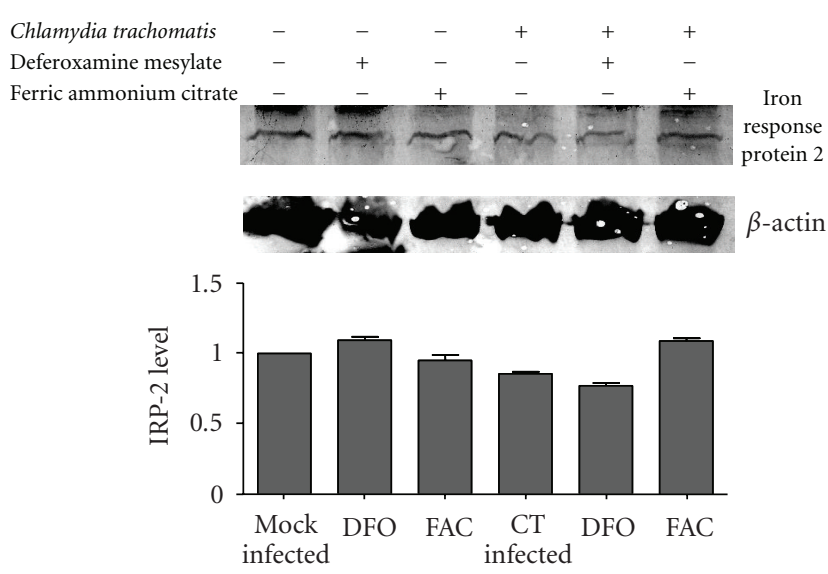

(b)

FIGURE 2: Effect of CT infection on IRP-1 expression in HeLa cells: $40 \mu \mathrm{g}$ cell lysates were electrophoresed on 8\% SDS-PAGE gels, followed by Western blotting using anti-IRP-1 and anti-IRP-2 antibodies, with beta-actin as control. (a) CT-infected HeLa cells showing higher expression of IRP-1, which declined after addition of DFX. (b) Decline in the level of IRP-2 expression was observed in CT-infected HeLa cells, and there was further decline consequent to addition of DFX. No significant change was observed in FAC-treated control and infected cells. Bars represent standard error. ${ }^{*} P<.05$ and ${ }^{* * *} P<.001$.

3.2. Expression of FHC in CT-Infected HeLa Cells. Chlamydia-infected HeLa cells showed higher expression of FHC in comparison to the mock-infected cells. Following the addition of DFX, FHC expression remained above the basal (mock) level. Moreover, subsequent to addition of FAC, upregulation of FHC was observed in both infected and mock-infected HeLa cells, as visualized by the Western blot assay (Figure 1(b)).
3.3. Levels of IRP-1 and IRP-2. In chlamydia-infected HeLa cells, the level of IRP-1 was higher in comparison to mockinfected cells (Figure 2(a)), whereas IRP-2 did not show any significant difference (Figure 2(b)). Consequent to the addition of the intracellular iron chelator DFX, the level of IRP-1 increased significantly, whereas IRP-2 did not show such change in CT-infected cells in comparison to mockinfected cells (Figure 2(b)). Addition of iron supplement led 


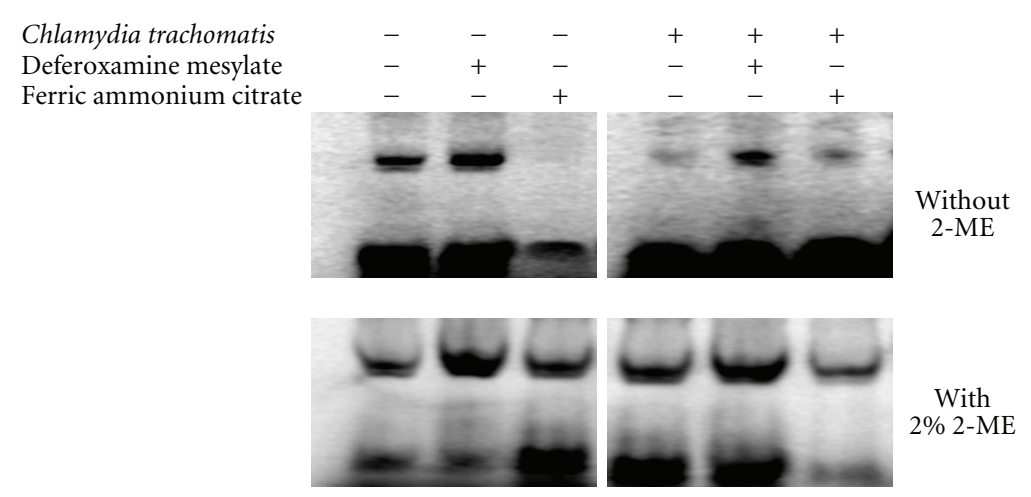

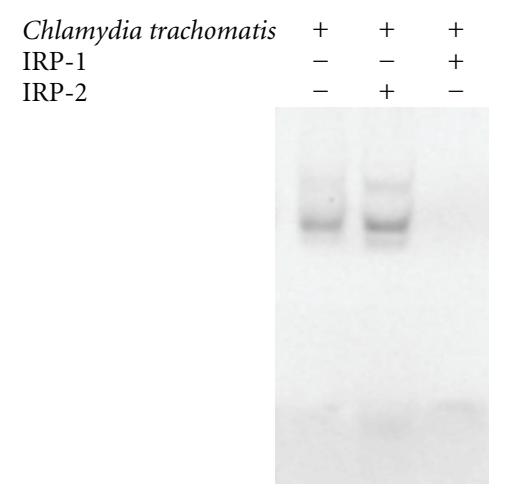

(b)

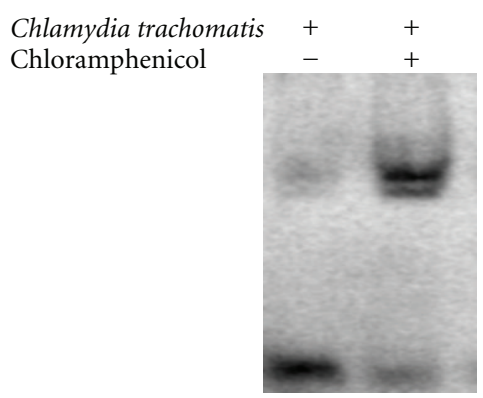

(c)

FIGURE 3: Effect of CT infection on the interaction of IRP-IRE: cytoplasmic extracts were prepared from CT-infected HeLa cells treated with DFX and FAC, along with their respective controls, at 24 hpi. Furthermore, the extracts were incubated with ${ }^{32} \mathrm{P}-\mathrm{labeled} \mathrm{RNA}$ probe, which contained the IRE sequence, in binding buffer. For the supershift assay, the corresponding antibodies (IRP-1 and IRP-2) were added. In parallel experiments, samples were treated with 2-mercaptoethanol) for determination of the maximum binding activity. (a) Binding activity of IRP-1/IRE was attenuated in CT-infected HeLa cells as observed in electrophoretic mobility-shift assay. (b) Inhibition of binding was observed with IRP-1 antibodies in CT-infected HeLa cells. (c) Attenuation of binding activity reverted to normal after treatment with chloramphenicol.

to significant changes in both infected and mock-infected HeLa cells (Figure 2(a)).

3.4. Attenuated Binding Activity of IRP-1/IRE (RNA Electrophoretic Mobility-Shift Assay). The binding activity was determined using the electrophoretic mobility-shift assay by comparison of the maximum activity (2 ME-treated cytoplasmic extract showed maximum activity) with the actual observed activity. The cytoplasmic extract of CTinfected HeLa cells showed a significant decrease in the binding activity relative to mock-infected cells. In the presence of DFX, increased binding activity was observed in both the conditions although the signal was relatively low in CT-infected cells (Figure 3(a)). When the cytosolic extract was preincubated with anti-IRP1 and anti-IRP2 antibodies, it showed loss of binding only in the case of IRP-1 (Figure 3(b)). Binding activity resumed in CT-infected HeLa cells after chloramphenicol treatment (Figure 3(c)).

\section{Discussion}

$\mathrm{CT}$ is one of the most successful bacterial pathogen in terms of intracellular survival due to its ability to modulate the host resources toward its own development. Both host and the pathogens have higher affinities for iron, the most essential element required for survival.

In accordance with previous studies, the current results showed a decline in the expression of TfR in CT-infected HeLa cells. In contrast to other intracellular pathogens, $C T$ showed upregulation of TfR expression [18]. TfR-mediated iron acquisition has been reported as an important mode of iron uptake, contributing the major part of the iron requirement in various cell types [19]. Regulation of TfR is sensitive to various intracellular physiological conditions; secretory factors, such as IFN- $\gamma$, tumor necrosis factor- $\alpha$, interleukin-1, and interleukin-6, downregulate the TfR level in various cell types [20]. Experimental models of chlamydial infection have shown upregulation in the levels of IFN- $\gamma$ [21]. Therefore, these results may suggest the involvement of secretory factors in the downregulation of TfR in chlamydiainfected cell. Furthermore, the present study showed that TfR expression did not change significantly after addition of DFX and FAC. This result deciphers the modulation of the iron-regulatory machinery of cells infected with CT.

In this study, upregulation of FHC was observed in CTinfected cells, which did not alter significantly after addition of the iron chelator DFX. Physiological responses reported 


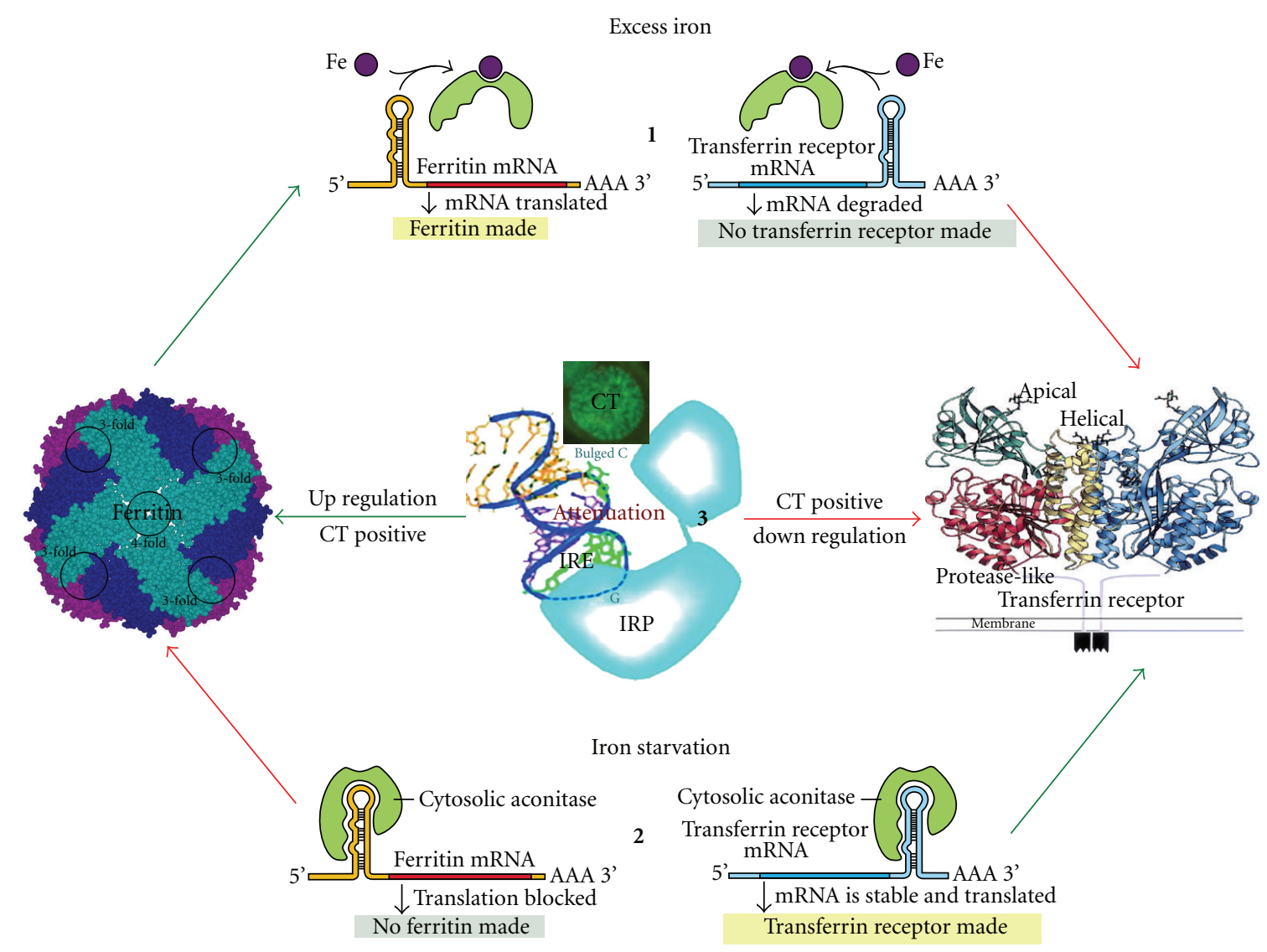

FIGURE 4: Translational initiation of ferritin and TfR mRNA degradation are regulated by binding of IRP to IRE in response to availability of iron. (1) In the excess iron, IRP forms an open conformation and is not able to bind IRE, subsequently translation of ferritin gets initiated and degradation of exposed TfR mRNA takes place. (2) In iron starved cells, IRP attains a closed conformation and binds to IRE present on 5' UTR of ferritin mRNA thus interferes with translation initiation and simultaneously stabilize TfR mRNA by binding 3' UTR. (3) Binding affinity of IRP-IRE is attenuated in presence of chlamydia leading to lower expression of TfR and higher expression of FHC [27].

for TNF- $\alpha$, IL-1, IL-6, and IFN- $\gamma$ include stimulation of $\mathrm{H}-$ ferritin expression and inhibition of TfR1 expression [19]. These results further explain the involvement of cytokines of the innate immune system in the upregulation of FHC. However, nonresponsiveness of the CT-infected cells to the iron chelator DFX might indicate modulation of the ironregulatory system.

Chlamydia-infected HeLa cells showed the predominant expression of IRP-1 rather than IRP-2, in contrast to mockinfected cells. In iron-depleted condition, IRP-1/2 binding to IRE blocks ferritin-mRNA translation and simultaneously stabilizes the TfR mRNA, thus regulating the iron uptake and storage [22]. Because of the additional 73 amino-acid peptide sequence, IRP-2 is prone to proteosomal degradation in the presence of nitric oxide and iron [23]. Chlamydial infection induces the production of both nitric oxide and IFN- $\gamma / \alpha$ in different cell types [24]. In response to the same stimulus, IRP-1 and -2 are regulated conversely [25]. Hence, it may suggest that lower levels of IRP-2 might be due to the proteosomal degradation induced by nitric oxide or converse regulation by IFN- $\gamma$.
In this study, Chlamydia-infected HeLa cells showed attenuation in IRP-IRE binding activity; however, the binding activity resumed after addition of chloramphenicol. Levels of TfR and ferritin are principally regulated through the binding of IRPs with IREs, and the binding activity of IRP-IRE is dependent on both the free reactive iron (labile iron pool) and the intracellular redox state [13]. The labile iron pool regulates the binding of IRP-1/2 to IREs through distinct mechanisms. In the presence of high levels of iron, IRP-1 assembles in a cubane [4Fe-4S] cluster, inhibiting the IRE-binding activity, and converting IRP-1 to an aconitase. When cellular iron stress is low, IRP-1 binds to IRE targets as an apoprotein (see Figure 4) [26, 27]. IRPs are also controlled by other effectors that include reactive oxygen species, which induce the disassembly of the Fe-S cluster of IRP-1 [28], and nitric oxide and hypoxia, which affect both IRPs [23].

Serine phosphorylation is an iron-independent mechanism of IRP-1 regulation, which destabilizes the Fe-S cluster $[29,30]$. The attenuated binding activity of IRP-1 leads to upregulation of FHC in Chlamydia-infected cells, in comparison to mock-infected cells, which is inhibited subsequent to DFX treatment. These results indicate that the 
level of intracellular free iron (Labile Iron Pool) is maintained at a critically functional state in chlamydia-infected cells; however, further study is required to establish this assumption. Iron restriction-induced chlamydial persistence is only achieved by DFX and not by exporter-dependent iron deprivation [31]. In a recent study, Nupur et al. have shown that Leishmania donovanii uses labile iron pool (LIP) as a dynamic source of iron inside the cells [32]. These observations further extend the possible involvement of LIP as a source of iron for intracellular development of CT. Hence, this study showed that chlamydial infection has a modulatory effect on the activity of IRPs which leads to decreased TfR and increased FHC expressions thus limiting the intracellular availability of catalytic-free iron and finally reactive oxygen species. Furthermore, chlamydial infection may accelerate the recycling of TfR and transferrin to the surface, thus recharge the required iron in LIP by faster shuttling. Transiting iron in LIP may be sequestered in FHC as stored available iron for CT in HeLa infected cells. Thus, the current findings suggest that attenuation in the binding activity of IRP-1/IRE may be one of the mechanisms involved in modulation of iron homeostasis in CT-infected cells.

\section{Acknowledgments}

The authors wish to thank the Indian Council of Medical Research (ICMR, India) for financial assistance to $\mathrm{H}$. Vardhan and R. Jha in the form of fellowship, and to the University Grant commission (UGC) India for providing fellowship to A. R. Bhengraj. This study was funded by Indo-US grants of Defense Research and Development Organization, India (DLS/RD-81/48222/LSRB 51/2003).

\section{References}

[1] J. W. Moulder, "Interaction of chlamydiae and host cells in vitro," Microbiological Reviews, vol. 55, no. 1, pp. 143-190, 1991.

[2] R. C. Brunham, B. Binns, F. Guijon, et al., "Etiology and outcome of acute pelvic inflammatory disease," Journal of Infectious Diseases, vol. 158, no. 3, pp. 510-517, 1988.

[3] T. Hackstadt, E. R. Fischer, M. A. Scidmore, D. D. Rockey, and R. A. Heinzen, "Origins and functions of the chlamydial inclusion," Trends in Microbiology, vol. 5, no. 7, pp. 288-293, 1997.

[4] W. L. Beatty, R. P. Morrison, and G. I. Byrne, "Persistent chlamydiae: from cell culture to a paradigm for chlamydial pathogenesis," Microbiological Reviews, vol. 58, no. 4, pp. 686699, 1994.

[5] R. J. Hogan, S. A. Mathews, S. Mukhopadhyay, J. T. Summersgill, and P. Timms, "Chlamydial persistence: beyond the biphasic paradigm," Infection and Immunity, vol. 72, no. 4, pp. 1843-1855, 2004.

[6] A. M. Coles, D. J. Reynolds, A. Harper, A. Devitt, and J. H. Pearce, "Low-nutrient induction of abnormal chlamydial development: a novel component of chlamydial pathogenesis?" FEMS Microbiology Letters, vol. 106, no. 2, pp. 193-200, 1993.

[7] J. E. Raulston, "Response of Chlamydia trachomatis serovar $\mathrm{E}$ to iron restriction vitro and evidence for iron-regulated chlamydial proteins," Infection and Immunity, vol. 65, no. 11, pp. 4539-4547, 1997.

[8] R. B. Clark, P. F. Schatzki, and H. P. Dalton, "Ultrastructural analysis of the effects of erythromycin on the morphology and developmental cycle of Chlamydia trachomatis HAR-13," Archives of Microbiology, vol. 133, no. 4, pp. 278-282, 1982.

[9] W. L. Beatty, T. A. Belanger, A. A. Desai, R. P. Morrison, and G. I. Byrne, "Tryptophan depletion as a mechanism of gamma interferon-mediated chlamydial persistence," Infection and Immunity, vol. 62, no. 9, pp. 3705-3711, 1994.

[10] R. J. Belland, D. E. Nelson, D. Virok, et al., "Transcriptome analysis of chlamydial growth during IFN- $\gamma$-mediated persistence and reactivation," Proceedings of the National Academy of Sciences of the United States of America, vol. 100, no. 26, pp. 15971-15976, 2003.

[11] J. T. Groves, "The bioinorganic chemistry of iron in oxygenases and supramolecular assemblies," Proceedings of the National Academy of Sciences of the United States of America, vol. 100, no. 7, pp. 3569-3574, 2003.

[12] K. Pantopoulos, "Iron metabolism and the IRE/IRP regulatory system: an update," Annals of the New York Academy of Sciences, vol. 1012, pp. 1-13, 2004.

[13] M. W. Hentze, M. U. Muckenthaler, and N. C. Andrews, "Balancing acts: molecular control of mammalian iron metabolism," Cell, vol. 117, no. 3, pp. 285-297, 2004.

[14] J. E. Raulston, "Response of Chlamydia trachomatis serovar E to iron restriction vitro and evidence for iron-regulated chlamydial proteins," Infection and Immunity, vol. 65, no. 11, pp. 4539-4547, 1997.

[15] J. E. Raulston, Chlamydia: Genomics \& Pathogenesis, Horizon Biosciences, Portland, Ore, USA, 2006.

[16] A. Rau, S. Wyllie, J. Whittimore, and J. E. Raulston, "Identification of Chlamydia trachomatis genomic sequences recognized by chlamydial divalent cation-dependent regulator A (DcrA)," Journal of Bacteriology, vol. 187, no. 2, pp. 443-448, 2005.

[17] M. A. Scidmore, "Cultivation and Laboratory Maintenance of Chlamydia trachomatis," Current Protocols in Microbiology, 2005.

[18] M. Gail, U. Gross, and W. Bohne, "Transcriptional profile of Toxoplasma gondii-infected human fibroblasts as revealed by gene-array hybridization," Molecular Genetics and Genomics, vol. 265, no. 5, pp. 905-912, 2001.

[19] F. M. Torti and S. V. Torti, "Regulation of ferritin genes and protein," Blood, vol. 99, no. 10, pp. 3505-3516, 2002.

[20] S. Ludwiczek, E. Aigner, I. Theurl, and G. Weiss, "Cytokinemediated regulation of iron transport in human monocytic cells," Blood, vol. 101, no. 10, pp. 4148-4154, 2003.

[21] H. W. Murray, A. M. Granger, and R. F. Teitelbaum, "Gamma interferon-activated human macrophages and Toxoplasma gondii, Chlamydia psittaci, and Leishmania donovani: antimicrobial role of limiting intracellular iron," Infection and Immunity, vol. 59, no. 12, pp. 4684-4686, 1991.

[22] M. W. Hentze, S. W. Caughman, J. L. Casey, et al., "A model for the structure and functions of iron-responsive elements," Gene, vol. 72, no. 1-2, pp. 201-208, 1988.

[23] D.-K. Kang, J. Jeong, S. K. Drake, N. B. Wehr, T. A. Rouault, and R. L. Levine, "Iron regulatory protein 2 as iron sensor: iron-dependent oxidative modification of cysteine," The Journal of Biological Chemistry, vol. 278, no. 17, pp. 14857-14864, 2003.

[24] A. Devitt, P. A. Lund, A. G. Morris, and J. H. Pearce, "Induction of alpha/beta interferon and dependent nitric oxide synthesis during Chlamydia trachomatis infection of 
McCoy cells in the absence of exogenous cytokine," Infection and Immunity, vol. 64, no. 10, pp. 3951-3956, 1996.

[25] C. Bouton, L. Oliveira, and J.-C. Drapier, "Converse modulation of IRP1 and IRP2 by immunological stimuli in murine RAW 264.7 macrophages," The Journal of Biological Chemistry, vol. 273, no. 16, pp. 9403-9408, 1998.

[26] R. S. Eisenstein and K. L. Ross, "Novel roles for iron regulatory proteins in the adaptive response to iron deficiency," Journal of Nutrition, vol. 133, supplement 2, no. 5, pp. 1510S-1516S, 2003.

[27] A. R. Tracey, "The role of iron regulatory proteins in mammalian iron homeostasis and disease," Nature Chemical Biology, vol. 2, pp. 406-414, 2006.

[28] K. Pantopoulos and M. W. Hentze, "Activation of iron regulatory protein-1 by oxidative stress in vitro," Proceedings of the National Academy of Sciences of the United States of America, vol. 95, no. 18, pp. 10559-10563, 1998.

[29] N. M. Brown, S. A. Anderson, D. W. Steffen, et al., "Novel role of phosphorylation in Fe-S cluster stability revealed by phosphomimetic mutations at Ser-138 of iron regulatory protein 1," Proceedings of the National Academy of Sciences of the United States of America, vol. 95, no. 26, pp. 15235-15240, 1998.

[30] C. Fillebeen, D. Chahine, A. Caltagirone, P. Segal, and K. Pantopoulos, "A phosphomimetic mutation at Ser-138 renders iron regulatory protein 1 sensitive to iron-dependent degradation," Molecular and Cellular Biology, vol. 23, no. 19, pp. 6973-6981, 2003.

[31] B. D. Dill and J. E. Raulston, "Examination of an inducible expression system for limiting iron availability during Chlamydia trachomatis infection," Microbes and Infection, vol. 9, no. 8, pp. 947-953, 2007.

[32] A. K. Das, S. Biswas, S. Solanki, and C. K. Mukhopadhyay, "Leishmania donovani depletes labile iron pool to exploit iron uptake capacity of macrophage for its intracellular growth," Cellular Microbiology, vol. 11, pp. 83-94, 2009. 

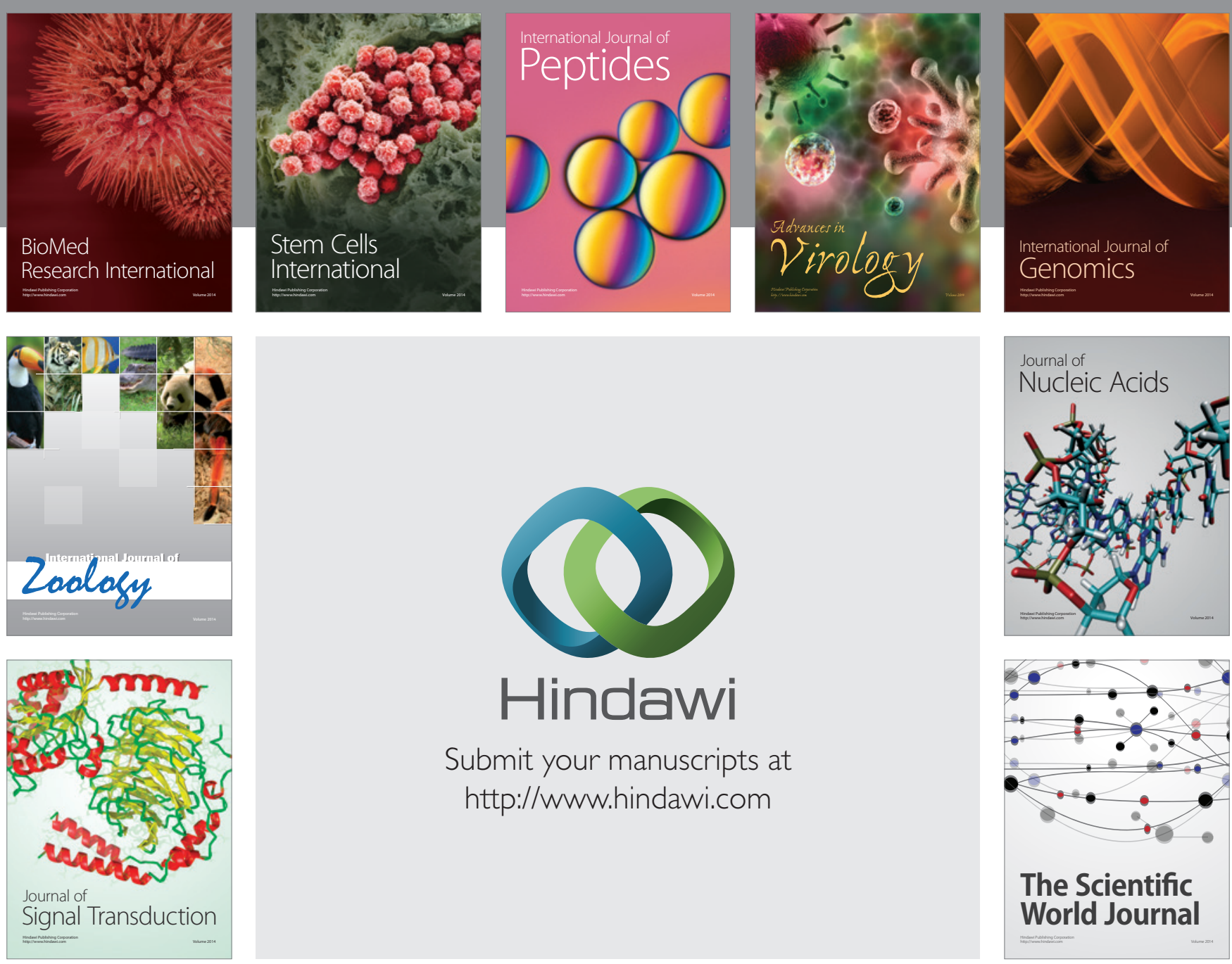

Submit your manuscripts at

http://www.hindawi.com
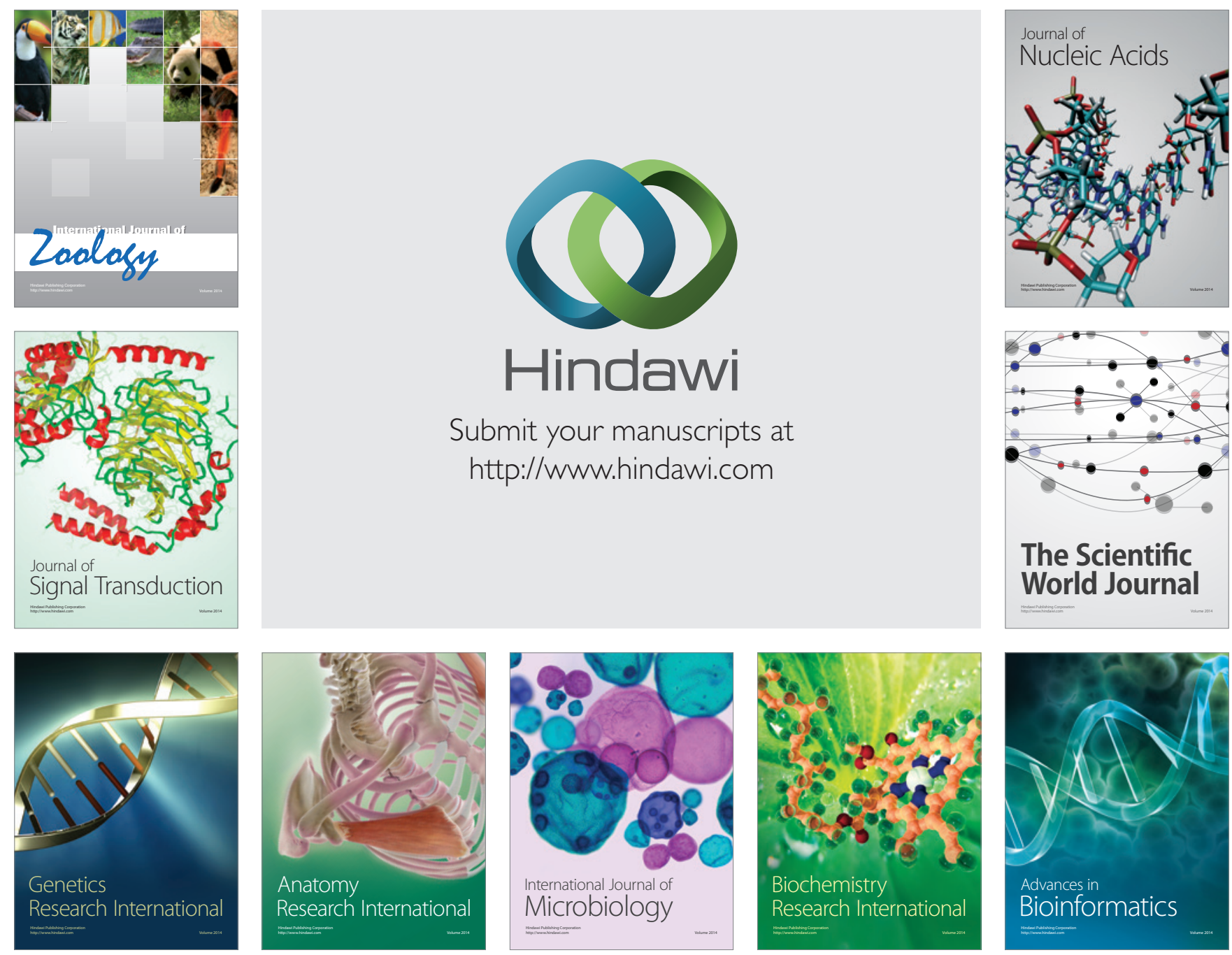

The Scientific World Journal
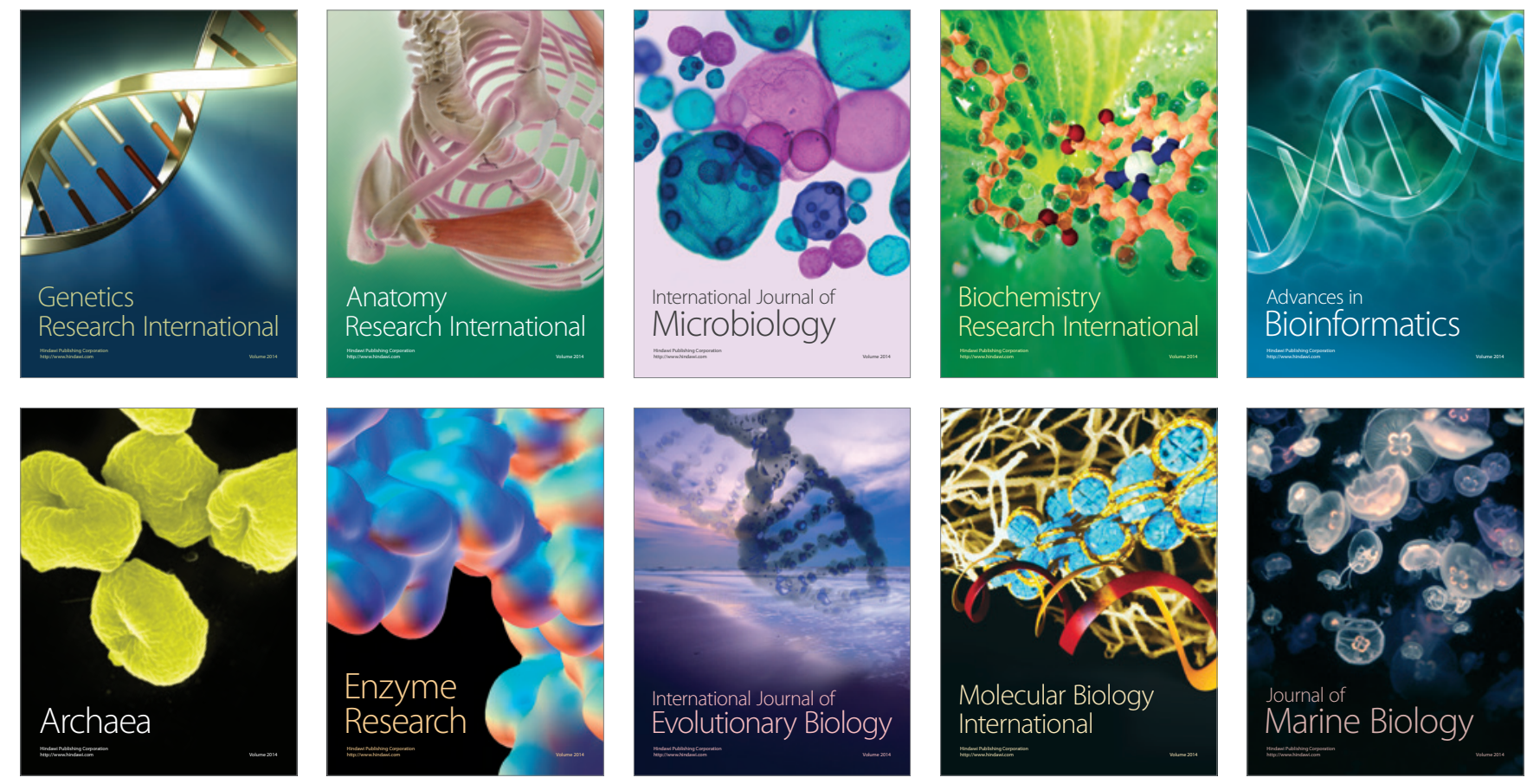